\title{
Some fixed point theorems for generalized $(\psi-\phi)$-weak contraction mappings in partial metric spaces
}

\author{
G.S. SAluJA
}

\begin{abstract}
The aim of this paper is to introduce the concepts of generalized $(\psi-\phi)$-weak contraction mappings of type $(A)$ and $(B)$ and establish some fixed point theorems for said contraction mappings in complete partial metric spaces. Our results extend and generalize several results from the current existing literature.
\end{abstract}

\section{INTRODUCTION}

The Banach contraction mappings principle is the opening and vital result in the direction of fixed point theory. In this theory, contraction is one of the main tools to prove the existence and uniqueness of a fixed point. Banach's contraction principle which gives an answer to the existence and uniqueness of a solution of an operator equation $T x=x$, is the most widely used fixed point theorem in all of analysis. This principle is constructive in nature and is one of the most useful techniques in the study of nonlinear equations. Banach contraction principle has been generalized in various ways either by using contractive conditions or by imposing some additional conditions on the underlying space.

In 1994, Matthews ([21, 22]) launched the notion of partial metric spaces as a generalizations of the notion of metric space in which, in definition of metric the condition $d(x, x)=0$ is replaced by the condition $d(x, x) \leq$ $d(x, y)$. In ([22]), Matthews discussed some properties of convergence of sequences and proved the fixed point theorem for contraction mapping on partial metric spaces: any mapping $S$ of a complete partial metric space $X$ onto itself that satisfies, where $0 \leq k<1$, the inequality $p(S(x), S(y)) \leq$ $k p(x, y)$ for all $x, y \in X$, has a unique fixed point. Also, the concept of PMS provides to study denotational semantics of dataflow networks $[21,22,27$, 31].

2010 Mathematics Subject Classification. 47H09, 47H10.

Key words and phrases. Fixed point, generalized $(\psi-\phi)$-weak contraction mapping, partial metric space.

Full paper. Received 8 July 2020, revised 18 August 2020, accepted 2 September 2020, available online 4 September 2020. 
Many authors studied the fixed points for mappings satisfying contractive conditions in complete partial metric spaces. More recently, in [3], [5], [6], [7], [11], [12], [18], [30] some fixed point theorems under various contractive conditions in complete partial metric spaces are proved.

Alber and Guerre-Delabriere [4], defined weakly contractive mappings on a Hilbert space and established a novel fixed point theorem for such a mappings. Subsequently, Rhoades [26] used the notion of weakly contractive mappings and obtained a fixed point theorem in complete metric space. Afterward, weak contraction and function satisfying weak contractive type inequalities have been considered in a large number of papers, (see, for instance [1], [2], [9], [13], [14], [15], [16], [23], [25], [28], [29] and references therein).

In this paper, we introduce the concept of generalized $(\psi-\phi)$-weak contraction mapping and establish some fixed point theorems for said class of mappings in complete partial metric spaces.

The main purpose of the present work is encouraged by its possible application, especially in discrete models for numerical analysis, where iterative schemes are extensively used due to their versatility for computer simulation. These models play an essential role in applied mathematical studies of certain nonlinear processes in relation with economics, biology and numerical physics.

\section{Definitions And Lemmas}

The definition of partial metric space is given by Matthews ([21]) as follows:

Definition 1 ([21]). Let $X$ be a nonempty set. A function $p: X \times X \rightarrow \mathbb{R}^{+}$ is said to be a partial metric on $X$ if for any $x, y, z \in X$, the following conditions hold:

$(P 1) x=y \Leftrightarrow p(x, x)=p(x, y)=p(y, y)$,

$(P 2) p(x, x) \leq p(x, y)$,

(P3) $p(x, y)=p(y, x)$,

(P4) $p(x, y) \leq p(x, z)+p(z, y)-p(z, z)$.

The pair $(X, p)$ is called a partial metric space (in short PMS).

If $p(x, y)=0$, then from $(P 1)$ and $(P 2), x=y$, but the converse does not always hold. Various applications of this space has been extensively investigated by many authors (see [20], [30] for details).

Remark 1 ([17]). Let $(X, p)$ be a partial metric space.

(1) The function $d_{p}: X \times X \rightarrow \mathbb{R}^{+}$defined as $d_{p}(x, y)=2 p(x, y)-$ $p(x, x)-p(y, y)$ is a (usual) metric on $X$ and $\left(X, d_{p}\right)$ is a (usual) metric space. 
(2) The function $d_{m}: X \times X \rightarrow \mathbb{R}^{+}$defined as $d_{m}(x, y)=\max \{p(x, y)-$ $p(x, x), p(x, y)-p(y, y)\}$ is a (usual) metric on $X$ and $\left(X, d_{m}\right)$ is a (usual) metric space.

It is clear that $d_{p}$ and $d_{m}$ are equivalent. Each partial metric $p$ on $X$ generates a $T_{0}$ topology $\tau_{p}$ on $X$ with a base of the family of open $p$-balls $\left\{B_{p}(x, \varepsilon): x \in X, \varepsilon>0\right\}$ where $B_{p}(x, \varepsilon)=\{y \in X: p(x, y) \leq p(x, x)+\varepsilon\}$ for all $x \in X$ and $\varepsilon>0$.

Example 1 ([7]). Let $X=\mathbb{R}^{+}$and $p: X \times X \rightarrow \mathbb{R}^{+}$given by $p(x, y)=$ $\max \{x, y\}$ for all $x, y \in \mathbb{R}^{+}$. Then $\left(\mathbb{R}^{+}, p\right)$ is a partial metric space.

Example $2([7])$. Let $X=\{[a, b]: a, b \in \mathbb{R}, a \leq b\}$. Then $p([a, b],[c, d])=$ $\max \{b, d\}-\min \{a, c\}$ defines a partial metric $p$ on $X$.

On a partial metric space the notions of convergence, the Cauchy sequence, completeness and continuity are defined as follows [21].

Definition 2 ([21]). Let $(X, p)$ be a partial metric space.

a) A sequence $\left\{x_{n}\right\}$ in $(X, p)$ is said to be convergent to a point $x \in X$ if and only if $p(x, x)=\lim _{n \rightarrow \infty} p\left(x_{n}, x\right)$,

b) A sequence $\left\{x_{n}\right\}$ is called a Cauchy sequence if $\lim _{m, n \rightarrow \infty} p\left(x_{m}, x_{n}\right)$ exists and finite,

c) $(X, p)$ is said to be complete if every Cauchy sequence $\left\{x_{n}\right\}$ in $X$ converges to a point $x \in X$ with respect to $\tau_{p}$. Furthermore,

$$
\lim _{m, n \rightarrow \infty} p\left(x_{m}, x_{n}\right)=\lim _{n \rightarrow \infty} p\left(x_{n}, x\right)=p(x, x) .
$$

d) A mapping $f: X \rightarrow X$ is said to be continuous at $x_{0} \in X$ if for every $\varepsilon>0$, there exists $\delta>0$ such that $f\left(B_{p}\left(x_{0}, \delta\right)\right) \subset B_{p}\left(f\left(x_{0}\right), \varepsilon\right)$.

Definition 3 ([24]). Let $(X, p)$ be a partial metric space.

(1) A sequence $\left\{x_{n}\right\}$ in $(X, p)$ is called 0-Cauchy if $\lim _{m, n \rightarrow \infty} p\left(x_{m}, x_{n}\right)=$ 0

(2) $(X, p)$ is said to be 0 -complete if every 0-Cauchy sequence $\left\{x_{n}\right\}$ in $X$ converges to a point $x \in X$, such that $p(x, x)=0$.

Lemma 1 ([21, 22]). Let $(X, p)$ be a partial metric space.

(a1) A sequence $\left\{x_{n}\right\}$ in $(X, p)$ is a Cauchy sequence if and only if it is a Cauchy sequence in the metric space $\left(X, d_{p}\right)$,

(a2) $(X, p)$ is complete if and only if the metric space $\left(X, d_{p}\right)$ is complete,

(a3) A subset $E$ of a partial metric space $(X, p)$ is closed if a sequence $\left\{x_{n}\right\}$ in $E$ such that $\left\{x_{n}\right\}$ converges to some $x \in X$, then $x \in E$.

Lemma 2 ([3]). Assume that $x_{n} \rightarrow x$ as $n \rightarrow \infty$ in a partial metric space $(X, p)$ such that $p(x, x)=0$. Then $\lim _{n \rightarrow \infty} p\left(x_{n}, y\right)=p(x, y)$ for every $y \in X$. 
Definition 4 ([4], Weak Contraction Mapping). Let $(X, d)$ be a complete metric space. A mapping $f: X \rightarrow X$ is said to be weakly contractive if

$$
d(f(x), f(y)) \leq d(x, y)-\psi(d(x, y)),
$$

where $x, y \in X, \psi:[0, \infty) \rightarrow[0, \infty)$ is continuous and non-decreasing, $\psi(x)=0$ if and only if $x=0$ and $\lim _{x \rightarrow \infty} \psi(x)=\infty$.

If we take $\psi(x)=c x$ where $0<c<1$ then it reduces to the Banach contraction condition given in [8].

Definition $5([19])$. The function $\phi:[0,+\infty) \rightarrow[0,+\infty)$ is called an altering distance function if the following properties are satisfied:

(1) $\phi$ is continuous and nondecreasing.

(2) $\phi(t)=0$ if and only if $t=0$.

\section{Main Results}

In this section, we shall establish some unique fixed point theorem in a complete partial metric space. We begin with the following.

Let $(X, p)$ be a partial metric space and $\mathcal{T}: X \rightarrow X$ be a mapping. We set for all $x, y \in X$

$$
\begin{aligned}
& \Theta(x, y)=\max \{p(x, y), p(x, \mathcal{T} x), p(y, \mathcal{T} y),\left.\frac{1}{3}[p(x, \mathcal{T} y)+p(y, \mathcal{T} y)+p(y, \mathcal{T} x)]\right\}, \\
& \theta(x, y)=\max \{p(x, y), p(y, \mathcal{T} y)\} .
\end{aligned}
$$

With the above setting, we introduce the following definition.

Definition 6. Let $(X, p)$ be a partial metric space and $\psi, \phi$ be altering distance functions. A mapping $\mathcal{T}: X \rightarrow X$ is called a generalized $(\psi-\phi)$ weak contraction of type $(A)$ if for any $x, y \in X$, we have

$$
\psi(p(\mathcal{T} x, \mathcal{T} y)) \leq \psi(\Theta(x, y))-\phi(\theta(x, y)) .
$$

Now, we prove our main result.

Theorem 1. Let $(X, p)$ be a complete partial metric space and let $\mathcal{T}: X \rightarrow$ $X$ be a generalized $(\psi-\phi)$-weak contraction of type $(A)$ mapping (4). Then $\mathcal{T}$ has a unique fixed point.

Proof. Let $x_{0} \in X$. We define a sequence $\left\{x_{n}\right\}$ in $X$ such that

$$
x_{n+1}=\mathcal{T} x_{n}, \quad \text { for any } n \in \mathbb{N}
$$

If for some $n \in \mathbb{N}, x_{n+1}=x_{n}$, then $x_{n}=\mathcal{T} x_{n}$, that is, $\mathcal{T}$ has a fixed point. Thus, we may assume that $x_{n+1} \neq x_{n}$ for all $n \in \mathbb{N}$. Now, using equation 
(4) and condition (P4), we have

$$
\begin{aligned}
\psi\left(p\left(x_{n}, x_{n+1}\right)\right) & =\psi\left(p\left(\mathcal{T} x_{n-1}, \mathcal{T} x_{n}\right)\right) \\
& \leq \psi\left(\Theta\left(x_{n-1}, x_{n}\right)\right)-\phi\left(\theta\left(x_{n-1}, x_{n}\right)\right),
\end{aligned}
$$

where

$$
\begin{aligned}
& \Theta\left(x_{n-1}, x_{n}\right)= \max \left\{p\left(x_{n-1}, x_{n}\right), p\left(x_{n-1}, \mathcal{T} x_{n-1}\right), p\left(x_{n}, \mathcal{T} x_{n}\right),\right. \\
&\left.\frac{1}{3}\left[p\left(x_{n-1}, \mathcal{T} x_{n}\right)+p\left(x_{n}, \mathcal{T} x_{n}\right)+p\left(x_{n}, \mathcal{T} x_{n-1}\right)\right]\right\} \\
&= \max \left\{p\left(x_{n-1}, x_{n}\right), p\left(x_{n-1}, x_{n}\right), p\left(x_{n}, x_{n+1}\right),\right. \\
&\left.\frac{1}{3}\left[p\left(x_{n-1}, x_{n+1}\right)+p\left(x_{n}, x_{n+1}\right)+p\left(x_{n}, x_{n}\right)\right]\right\} \\
& \leq \max \left\{p\left(x_{n-1}, x_{n}\right), p\left(x_{n-1}, x_{n}\right), p\left(x_{n}, x_{n+1}\right),\right. \\
&\left.\frac{1}{3}\left[p\left(x_{n-1}, x_{n}\right)+2 p\left(x_{n}, x_{n+1}\right)\right]\right\}, \\
& \theta\left(x_{n-1}, x_{n}\right)=\max \left\{p\left(x_{n-1}, x_{n}\right), p\left(x_{n}, \mathcal{T} x_{n}\right)\right\} \\
&=\max \left\{p\left(x_{n-1}, x_{n}\right), p\left(x_{n}, x_{n+1}\right)\right\} .
\end{aligned}
$$

From (6)-(7), we obtain

$$
\begin{aligned}
\psi\left(p\left(x_{n}, x_{n+1}\right)\right) \leq & \psi\left(\operatorname { m a x } \left\{p\left(x_{n-1}, x_{n}\right), p\left(x_{n-1}, x_{n}\right), p\left(x_{n}, x_{n+1}\right),\right.\right. \\
& \left.\left.\frac{1}{3}\left[p\left(x_{n-1}, x_{n}\right)+2 p\left(x_{n}, x_{n+1}\right)\right]\right\}\right) \\
& -\phi\left(\max \left\{p\left(x_{n-1}, x_{n}\right), p\left(x_{n}, x_{n+1}\right)\right\}\right) .
\end{aligned}
$$

Since

$$
\frac{1}{3}\left[p\left(x_{n-1}, x_{n}\right)+2 p\left(x_{n}, x_{n+1}\right)\right] \leq \max \left\{p\left(x_{n-1}, x_{n}\right), p\left(x_{n}, x_{n+1}\right)\right\},
$$

if for some $n \in \mathbb{N}$

$$
\max \left\{p\left(x_{n-1}, x_{n}\right), p\left(x_{n}, x_{n+1}\right)\right\}=p\left(x_{n}, x_{n+1}\right),
$$

then

$$
\psi\left(p\left(x_{n}, x_{n+1}\right)\right) \leq \psi\left(p\left(x_{n}, x_{n+1}\right)\right)-\phi\left(p\left(x_{n}, x_{n+1}\right)\right) .
$$

Thus, $\phi\left(p\left(x_{n}, x_{n+1}\right)\right)=0$, and, hence, by the property of $\phi, p\left(x_{n}, x_{n+1}\right)=$ 0 . Therefore, from from $(P 1)$ and $(P 2)$, we have $x_{n}=x_{n+1}$, which is a contradiction. So,

$$
\max \left\{p\left(x_{n-1}, x_{n}\right), p\left(x_{n}, x_{n+1}\right)\right\}=p\left(x_{n-1}, x_{n}\right),
$$


thus, we get

$$
\begin{gathered}
p\left(x_{n}, x_{n+1}\right) \leq p\left(x_{n-1}, x_{n}\right), \quad \forall n \in \mathbb{N} \\
\psi\left(p\left(x_{n}, x_{n+1}\right)\right) \leq \psi\left(p\left(x_{n-1}, x_{n}\right)\right)-\phi\left(p\left(x_{n-1}, x_{n}\right)\right), \quad \forall n \in \mathbb{N} .
\end{gathered}
$$

By (13), $\left\{p\left(x_{n}, x_{n+1}\right): n \in \mathbb{N}\right\}$ is a non-increasing sequence. Hence it is convergent to a real number, therefore there exists $c \geq 0$ such that

$$
\lim _{n \rightarrow \infty} p\left(x_{n}, x_{n+1}\right)=c .
$$

Taking the limit as $n \rightarrow \infty$ in (14), we obtain

$$
\psi(c) \leq \psi(c)-\phi(c) \text {. }
$$

Therefore $\phi(c)=0$ and, hence, $c=0$. Thus

$$
\lim _{n \rightarrow \infty} p\left(x_{n}, x_{n+1}\right)=0 \text {. }
$$

Now, we show that $\left\{x_{n}\right\}$ is a Cauchy sequence in $X$. If otherwise, then there exists $\varepsilon>0$ and increasing sequences of integers $\{m(k)\}$ and $\{n(k)\}$ such that for all integers $k$,

$$
\begin{gathered}
n(k)>m(k)>k, \\
p\left(x_{m(k)}, x_{n(k)}\right) \geq \varepsilon .
\end{gathered}
$$

Further corresponding to $m(k)$, we can choose $n(k)$ in such a way that it is the smallest integer with $n(k)>m(k)$ and satisfying (18). Then

$$
p\left(x_{m(k)}, x_{n(k)-1}\right)<\varepsilon .
$$

Now, using (19), (P3) and (P4), we have

$$
\begin{aligned}
\varepsilon & \leq p\left(x_{m(k)}, x_{n(k)}\right) \\
& \leq p\left(x_{m(k)}, x_{n(k)-1}\right)+p\left(x_{n(k)-1}, x_{n(k)}\right)-p\left(x_{n(k)-1}, x_{n(k)-1}\right) \\
& \leq p\left(x_{m(k)}, x_{n(k)-1}\right)+p\left(x_{n(k)-1}, x_{n(k)}\right) \\
& \leq \varepsilon+p\left(x_{n(k)-1}, x_{n(k)}\right), \quad(\text { by }(20)) .
\end{aligned}
$$

Letting $k \rightarrow+\infty$ in equation (21) and using (17), we get

$$
\lim _{k \rightarrow \infty} p\left(x_{m(k)}, x_{n(k)}\right)=\varepsilon \text {. }
$$

Again, with the help of (P3), (P4) and using (17), we have

$$
\begin{aligned}
p\left(x_{m(k)}, x_{n(k)}\right) \leq & p\left(x_{m(k)}, x_{m(k)-1}\right)+p\left(x_{n(k)}, x_{m(k)-1}\right) \\
& -p\left(x_{m(k)-1}, x_{m(k)-1}\right) \\
\leq & p\left(x_{m(k)}, x_{m(k)-1}\right)+p\left(x_{n(k)}, x_{m(k)-1}\right) \\
\leq & p\left(x_{n(k)}, x_{m(k)-1}\right) \\
\leq & p\left(x_{n(k)}, x_{n(k)-1}\right)+p\left(x_{n(k)-1}, x_{m(k)-1}\right) \\
& -p\left(x_{n(k)-1}, x_{n(k)-1}\right) \\
\leq & p\left(x_{m(k)-1}, x_{n(k)-1}\right) .
\end{aligned}
$$


Also, with the help of $(P 3),(P 4)$ and using (17), we have

$$
\begin{aligned}
p\left(x_{m(k)-1}, x_{n(k)-1}\right) \leq & p\left(x_{m(k)-1}, x_{m(k)}\right)+p\left(x_{n(k)-1}, x_{m(k)}\right) \\
& -p\left(x_{m(k)}, x_{m(k)}\right) \\
\leq & p\left(x_{m(k)-1}, x_{m(k)}\right)+p\left(x_{n(k)-1}, x_{m(k)}\right) .
\end{aligned}
$$

Letting $k \rightarrow+\infty$ in equation (24) and using (17), (22) and (23), we get

$$
\lim _{k \rightarrow \infty} p\left(x_{m(k)-1}, x_{n(k)-1}\right)=\varepsilon .
$$

Again note that with the help of $(P 3),(P 4)$ and using (17), we have

$$
\begin{aligned}
p\left(x_{m(k)}, x_{n(k)}\right) \leq & p\left(x_{m(k)}, x_{m(k)-1}\right)+p\left(x_{n(k)}, x_{m(k)-1}\right) \\
& -p\left(x_{m(k)-1}, x_{m(k)-1}\right) \\
\leq & p\left(x_{m(k)}, x_{m(k)-1}\right)+p\left(x_{m(k)-1}, x_{n(k)}\right) \\
\leq & p\left(x_{m(k)-1}, x_{n(k)}\right) .
\end{aligned}
$$

Again note that

$$
\begin{aligned}
p\left(x_{m(k)-1}, x_{n(k)}\right) \leq & p\left(x_{m(k)-1}, x_{m(k)}\right)+p\left(x_{m(k)}, x_{n(k)}\right) \\
& -p\left(x_{m(k)}, x_{m(k)}\right) \\
\leq & p\left(x_{m(k)-1}, x_{m(k)}\right)+p\left(x_{m(k)}, x_{n(k)}\right) .
\end{aligned}
$$

Letting $k \rightarrow+\infty$ in equation (27) and using (17), (22) and (26), we get

$$
\lim _{k \rightarrow \infty} p\left(x_{m(k)-1}, x_{n(k)}\right)=\varepsilon .
$$

Again note that with the help of $(P 3),(P 4)$ and using (17), we have

$$
\begin{aligned}
p\left(x_{m(k)}, x_{n(k)}\right) \leq & p\left(x_{n(k)}, x_{n(k)-1}\right)+p\left(x_{n(k)-1}, x_{m(k)}\right) \\
& -p\left(x_{n(k)-1}, x_{n(k)-1}\right) \\
\leq & p\left(x_{n(k)}, x_{n(k)-1}\right)+p\left(x_{n(k)-1}, x_{m(k)}\right) \\
\leq & p\left(x_{n(k)-1}, x_{m(k)}\right) .
\end{aligned}
$$

Again note that

$$
\begin{aligned}
p\left(x_{n(k)-1}, x_{m(k)}\right) \leq & p\left(x_{n(k)-1}, x_{n(k)}\right)+p\left(x_{n(k)}, x_{m(k)}\right) \\
& -p\left(x_{n(k)}, x_{n(k)}\right) \\
\leq & p\left(x_{n(k)-1}, x_{n(k)}\right)+p\left(x_{n(k)}, x_{m(k)}\right) .
\end{aligned}
$$

Letting $k \rightarrow+\infty$ in equation (30) and using (17), (22) and (29), we get

$$
\lim _{k \rightarrow \infty} p\left(x_{n(k)-1}, x_{m(k)}\right)=\varepsilon .
$$

Now consider inequality (4) and putting $x=x_{m(k)-1}$ and $y=x_{n(k)-1}$, we obtain

$$
\begin{aligned}
\psi\left(p\left(x_{m(k)}, x_{n(k)}\right)\right)= & \psi\left(p\left(\mathcal{T} x_{m(k)-1}, \mathcal{T} x_{n(k)-1}\right)\right) \\
\leq & \psi\left(\Theta\left(x_{m(k)-1}, x_{n(k)-1}\right)\right) \\
& -\phi\left(\theta\left(x_{m(k)-1}, x_{n(k)-1}\right)\right)
\end{aligned}
$$


where

$$
\begin{aligned}
& \Theta\left(x_{m(k)-1}, x_{n(k)-1}\right)=\max \left\{p\left(x_{m(k)-1}, x_{n(k)-1}\right), p\left(x_{m(k)-1}, \mathcal{T} x_{m(k)-1}\right),\right. \\
& p\left(x_{n(k)-1}, \mathcal{T} x_{n(k)-1}\right), \\
& \frac{1}{3}\left[p\left(x_{m(k)-1}, \mathcal{T} x_{n(k)-1}\right)+p\left(x_{n(k)-1}, \mathcal{T} x_{n(k)-1}\right)\right. \\
& \left.\left.+p\left(x_{n(k)-1}, \mathcal{T} x_{m(k)-1}\right)\right]\right\} \\
& =\max \left\{p\left(x_{m(k)-1}, x_{n(k)-1}\right), p\left(x_{m(k)-1}, x_{m(k)}\right),\right. \\
& p\left(x_{n(k)-1}, x_{n(k)}\right), \\
& \frac{1}{3}\left[p\left(x_{m(k)-1}, x_{n(k)}\right)+p\left(x_{n(k)-1}, x_{n(k)}\right)\right. \\
& \left.\left.+p\left(x_{n(k)-1}, x_{m(k)}\right)\right]\right\} \\
& \theta\left(x_{m(k)-1}, x_{n(k)-1}\right)=\max \left\{p\left(x_{m(k)-1}, x_{n(k)-1}\right), p\left(x_{n(k)-1}, \mathcal{T} x_{n(k)-1}\right)\right\} \\
& =\max \left\{p\left(x_{m(k)-1}, x_{n(k)-1}\right), p\left(x_{n(k)-1}, x_{n(k)}\right)\right\} .
\end{aligned}
$$

Substituting in (32), we get

$$
\begin{aligned}
& \psi\left(p\left(x_{m(k)}, x_{n(k)}\right)=\psi\left(p\left(\mathcal{T} x_{m(k)-1}, \mathcal{T} x_{n(k)-1}\right)\right)\right. \\
& \leq \psi\left(\operatorname { m a x } \left\{p\left(x_{m(k)-1}, x_{n(k)-1}\right), p\left(x_{m(k)-1}, x_{m(k)}\right),\right.\right. \\
& p\left(x_{n(k)-1}, x_{n(k)}\right), \\
& \frac{1}{3}\left[p\left(x_{m(k)-1}, x_{n(k)}\right)+p\left(x_{n(k)-1}, x_{n(k)}\right)\right. \\
& \left.\left.\left.\quad+p\left(x_{n(k)-1}, x_{m(k)}\right)\right]\right\}\right) \\
& -\phi\left(\max \left\{p\left(x_{m(k)-1}, x_{n(k)-1}\right), p\left(x_{n(k)-1}, x_{n(k)}\right)\right\}\right) .
\end{aligned}
$$

Letting $k \rightarrow+\infty$ in equation (33) and using (17), (22), (25), (28), (31) and the continuity of $\psi$ and $\phi$, we have

$$
\psi(\varepsilon) \leq \psi(\varepsilon)-\phi(\varepsilon)<\psi(\varepsilon),
$$

which is a contradiction since $\varepsilon>0$. This shows that $\left\{x_{n}\right\}$ is a Cauchy sequence in $X$. Thus by Lemma 1 this sequence will also Cauchy in $\left(X, d_{p}\right)$. In addition, since $(X, p)$ is complete, $\left(X, d_{p}\right)$ is also complete. Thus there exists $u \in X$ such that $x_{n} \rightarrow u$ as $n \rightarrow+\infty$.

Moreover by Lemma 2,

$$
p(u, u)=\lim _{n \rightarrow \infty} p\left(u, x_{n}\right)=\lim _{n, m \rightarrow \infty} p\left(x_{n}, x_{m}\right)=0
$$


implies

$$
\lim _{n \rightarrow \infty} d_{p}\left(u, x_{n}\right)=0
$$

Now, we show that $u$ is a fixed point of $\mathcal{T}$. Notice that due to (35), we have $p(u, u)=0$. By using inequality (4), we get

$$
\begin{aligned}
\psi\left(p\left(x_{n+1}, \mathcal{T} u\right)\right. & =\psi\left(p\left(\mathcal{T} x_{n}, \mathcal{T} u\right)\right) \\
& \leq \psi\left(\Theta\left(x_{n}, u\right)\right)-\phi\left(\theta\left(x_{n}, u\right)\right)
\end{aligned}
$$

where

$$
\begin{aligned}
& \Theta\left(x_{n}, u\right)=\max \left\{p\left(x_{n}, u\right), p\left(x_{n}, \mathcal{T} x_{n}\right), p(u, \mathcal{T} u)\right.\left.\frac{1}{3}\left[p\left(x_{n}, \mathcal{T} u\right)+p(u, \mathcal{T} u)+p\left(u, \mathcal{T} x_{n}\right)\right]\right\} \\
&= \max \left\{p\left(x_{n}, u\right), p\left(x_{n}, x_{n+1}\right), p(u, \mathcal{T} u)\right. \\
&\left.\frac{1}{3}\left[p\left(x_{n}, \mathcal{T} u\right)+p(u, \mathcal{T} u)+p\left(u, x_{n+1}\right)\right]\right\}, \\
& \theta\left(x_{n}, u\right)=\max \left\{p\left(x_{n}, u\right), p(u, \mathcal{T} u)\right\} .
\end{aligned}
$$

Letting $n \rightarrow+\infty$ in the above and using (35), we get

$$
\begin{aligned}
\Theta\left(x_{n}, u\right) & \rightarrow p(u, \mathcal{T} u), \\
\theta\left(x_{n}, u\right) & \rightarrow p(u, \mathcal{T} u) .
\end{aligned}
$$

On letting $n \rightarrow+\infty$ in (37) and using the continuity of $\psi$ and $\phi$, we get

$$
\psi(p(u, \mathcal{T} u)) \leq \psi(p(u, \mathcal{T} u))-\phi(p(u, \mathcal{T} u))<\psi(p(u, \mathcal{T} u)),
$$

a contradiction. Therefore $\psi(p(u, \mathcal{T} u))=0$ and hence by the property of $\psi$, we have $p(u, \mathcal{T} u)=0$ and so $u=\mathcal{T} u$. Thus $u$ is a fixed point of $\mathcal{T}$.

Now, to show that the fixed point of $\mathcal{T}$ is unique. For this, suppose $u, v \in X$ be two fixed points of $\mathcal{T}$ such that $u \neq v$. Therefore from equation (4) and using (P3) and (35), we have

$$
\psi(p(u, v))=\psi(p(\mathcal{T} u, \mathcal{T} v)) \leq \psi(\Theta(u, v))-\phi(\theta(u, v)),
$$

where

$$
\begin{aligned}
\Theta(u, v)= & \max \{p(u, v), p(u, \mathcal{T} u), p(v, \mathcal{T} v), \\
& \left.\frac{1}{3}[p(u, \mathcal{T} v)+p(v, \mathcal{T} v)+p(v, \mathcal{T} u)]\right\} \\
= & \max \{p(u, v), p(u, u), p(v, v), \\
& \left.\frac{1}{3}[p(u, v)+p(v, v)+p(v, u)]\right\} \\
= & \max \left\{p(u, v), 0,0, \frac{2}{3} p(u, v)\right\} \\
= & p(u, v),
\end{aligned}
$$




$$
\begin{aligned}
\theta(u, v) & =\max \{p(u, v), p(v, \mathcal{T} v)\} \\
& =\max \{p(u, v), p(v, v)\} \\
& =\max \{p(u, v), 0\} \\
& =p(u, v)
\end{aligned}
$$

By (40) and (41), we obtain

$$
\begin{aligned}
\psi(p(u, v)) & =\psi(p(\mathcal{T} u, \mathcal{T} v)) \\
& \leq \psi(p(u, v))-\phi(p(u, v)) \\
& <\psi(p(u, v)),
\end{aligned}
$$

a contradiction. Thus $\psi(p(u, v))=0$ and hence $p(u, v)=0$ by the property of $\psi$ and therefore $u=v$. This shows that the fixed point of $\mathcal{T}$ is unique.

This completes the proof.

Corollary 1. Let $(X, p)$ be a complete partial metric space and let $\mathcal{T}: X \rightarrow$ $X$ be a mapping. Suppose there exists $k \in[0,1)$ such that

$$
\begin{aligned}
p(\mathcal{T} x, \mathcal{T} y) \leq k \max \{ & p(x, y), p(x, \mathcal{T} x), p(y, \mathcal{T} y), \\
& \left.\frac{1}{3}[p(x, \mathcal{T} y)+p(y, \mathcal{T} y)+p(y, \mathcal{T} x)]\right\},
\end{aligned}
$$

for all $x, y \in X$. Then $\mathcal{T}$ has a unique fixed point.

If we take

$\max \left\{p(x, y), p(x, \mathcal{T} x), p(y, \mathcal{T} y), \frac{1}{3}[p(x, \mathcal{T} y)+p(x, \mathcal{T} x)+p(y, \mathcal{T} x)]\right\}=p(x, y)$ in Corollary 1 , then we have the following result.

Corollary 2 ([22]). Let $(X, p)$ be a complete partial metric space and let $\mathcal{T}: X \rightarrow X$ be a mapping. Suppose there exists $k \in[0,1)$ such that

$$
p(\mathcal{T} x, \mathcal{T} y) \leq k p(x, y),
$$

for all $x, y \in X$. Then $\mathcal{T}$ has a unique fixed point.

Remark 2. Corollary 2 extends well-known Banach contraction mapping principle ([8]) from complete metric space to the setting of complete partial metric space.

Corollary 3. Let $(X, p)$ be a complete partial metric space and let $\mathcal{T}: X \rightarrow$ $X$ be a mapping. Suppose there exist nonnegative real numbers $\alpha, \beta, \gamma$ and $\delta$ with $\alpha+\beta+\gamma+\delta<1$ such that

$$
\begin{aligned}
p(\mathcal{T} x, \mathcal{T} y) \leq & \alpha p(x, y)+\beta p(x, \mathcal{T} x)+\gamma p(y, \mathcal{T} y) \\
& +\frac{\delta}{3}[p(x, \mathcal{T} y)+p(y, \mathcal{T} y)+p(y, \mathcal{T} x)],
\end{aligned}
$$

for all $x, y \in X$. Then $\mathcal{T}$ has a unique fixed point. 
Proof. Follows from Corollary 1, by using

$$
\begin{aligned}
& \alpha p(x, y)+\beta p(x, \mathcal{T} x)+\gamma p(y, \mathcal{T} y) \\
& +\frac{\delta}{3}[p(x, \mathcal{T} y)+p(y, \mathcal{T} y)+p(y, \mathcal{T} x)] \\
& \leq(\alpha+\beta+\gamma+\delta) \max \{p(x, y), p(x, \mathcal{T} x), p(y, \mathcal{T} y) \\
& \left.\quad \frac{1}{3}[p(x, \mathcal{T} y)+p(y, \mathcal{T} y)+p(y, \mathcal{T} x)]\right\} .
\end{aligned}
$$

Again we consider $(X, p)$ is a partial metric space, and $\mathcal{T}: X \rightarrow X$ a mapping. We set

$$
\begin{gathered}
\mathcal{M}(x, y)=\max \{p(x, y), p(x, \mathcal{T} x), p(y, \mathcal{T} y), \\
\left.\frac{1}{3}[p(x, \mathcal{T} y)+p(x, \mathcal{T} x)+p(y, \mathcal{T} x)]\right\}, \\
\mathcal{N}(x, y)=\max \{p(x, \mathcal{T} x), p(y, \mathcal{T} y)\} .
\end{gathered}
$$

Now, we introduce the following notion.

Definition 7. Let $(X, p)$ be a partial metric space and $\psi, \phi$ be altering distance functions. A mapping $\mathcal{T}: X \rightarrow X$ is called a generalized $(\psi-\phi)$ weak contraction of type $(B)$ if for any $x, y \in X$, we have

$$
\psi(p(\mathcal{T} x, \mathcal{T} y) \leq \psi(\mathcal{M}(x, y))-\phi(\mathcal{N}(x, y)) .
$$

Following the same arguments as those in the proof of Theorem 1, we obtain the following.

Theorem 2. Let $(X, p)$ be a complete partial metric space and let $\mathcal{T}: X \rightarrow$ $X$ be a generalized $(\psi-\phi)$-weak contraction of type $(B)$, that is, satisfying condition (49). Then $\mathcal{T}$ has a unique fixed point.

The following results are direct consequences of Theorem 2 .

Corollary 4. Let $(X, p)$ be a complete partial metric space and let $\mathcal{T}: X \rightarrow$ $X$ be a mapping. Suppose there exists $k \in[0,1)$ such that

$$
\begin{aligned}
p(\mathcal{T} x, \mathcal{T} y) \leq k \max & \{p(x, y), p(x, \mathcal{T} x), p(y, \mathcal{T} y), \\
& \left.\frac{1}{3}[p(x, \mathcal{T} y)+p(x, \mathcal{T} x)+p(y, \mathcal{T} x)]\right\},
\end{aligned}
$$

for all $x, y \in X$. Then $\mathcal{T}$ has a unique fixed point.

Corollary 5. Let $(X, p)$ be a complete partial metric space and let $\mathcal{T}: X \rightarrow$ $X$ be a mapping. Suppose there exist nonnegative real numbers $\alpha, \beta, \gamma$ and $\delta$ with $\alpha+\beta+\gamma+\delta<1$ such that

$$
\begin{aligned}
p(\mathcal{T} x, \mathcal{T} y) \leq & \alpha p(x, y)+\beta p(x, \mathcal{T} x)+\gamma p(y, \mathcal{T} y) \\
& +\frac{\delta}{3}[p(x, \mathcal{T} y)+p(x, \mathcal{T} x)+p(y, \mathcal{T} x)],
\end{aligned}
$$


for all $x, y \in X$. Then $\mathcal{T}$ has a unique fixed point.

We also obtain some fixed point theorems of integral type as corollaries to the results.

Denote $\Phi$ the set of functions $\phi:[0,+\infty) \rightarrow[0,+\infty)$ satisfying the following hypothesis:

(H1) $\phi$ is a Lebesgue-integrable mapping on each compact subset of $[0,+\infty)$;

(H2) for any $\varepsilon>0$ we have $\int_{0}^{\varepsilon} \phi(s) \mathrm{d} s>0$.

It is an easy matter, to see that the mapping $\psi:[0,+\infty) \rightarrow[0,+\infty)$ defined by

$$
\psi(t)=\int_{0}^{t} \phi(s) \mathrm{d} s
$$

an altering distance function. Now, we have the following result.

Corollary 6. Let $(X, p)$ be a complete partial metric space. Let $\mathcal{T}: X \rightarrow X$ be a mapping such that for $\phi, \lambda \in \Phi$, we have

$$
\begin{aligned}
& \int_{0}^{p(\mathcal{T} x, \mathcal{T} y)} \phi(s) \mathrm{d} s \\
& \leq \int_{0}^{\max \left\{p(x, y), p(x, \mathcal{T} x), p(y, \mathcal{T} y), \frac{1}{3}[p(x, \mathcal{T} y)+p(y, \mathcal{T} y)+p(y, \mathcal{T} x)]\right\}} \phi(s) \mathrm{d} s \\
& \quad-\int_{0}^{\max \{p(x, y), p(y, \mathcal{T} y)\}} \lambda(s) \mathrm{d} s
\end{aligned}
$$

for all $x, y \in X$. Then $\mathcal{T}$ has a unique fixed point.

Proof. Follows from Theorem 1 by taking

$$
\psi(t)=\int_{0}^{t} \phi(s) \mathrm{d} s, \quad \phi(t)=\int_{0}^{t} \lambda(s) \mathrm{d} s .
$$

Corollary 7. Let $(X, p)$ be a complete partial metric space. Let $\mathcal{T}: X \rightarrow X$ be a mapping such that for $\phi, \lambda \in \Phi$, we have

$$
\begin{aligned}
& \int_{0}^{p(\mathcal{T} x, \mathcal{T} y)} \phi(s) \mathrm{d} s \\
& \leq \int_{0}^{\max \left\{p(x, y), p(x, \mathcal{T} x), p(y, \mathcal{T} y), \frac{1}{3}[p(y, \mathcal{T} x)+p(x, \mathcal{T} x)+p(x, \mathcal{T} y)]\right\}} \phi(s) \mathrm{d} s \\
& \quad-\int_{0}^{\max \{p(x, \mathcal{T} x), p(y, \mathcal{T} y)\}} \lambda(s) \mathrm{d} s
\end{aligned}
$$

for all $x, y \in X$. Then $\mathcal{T}$ has a unique fixed point. 
Proof. Follows from Theorem 2 by taking

$$
\psi(t)=\int_{0}^{t} \phi(s) \mathrm{d} s, \quad \phi(t)=\int_{0}^{t} \lambda(s) \mathrm{d} s .
$$

The following result is a special case of Corollary 1 and 4 .

Corollary 8. Let $(X, p)$ be a complete partial metric space. Let $\mathcal{T}: X \rightarrow X$ be a mapping. Suppose that there exists $0<k<1$ such that for $\phi \in \Phi$, we have

$$
\int_{0}^{p(\mathcal{T} x, \mathcal{T} y)} \phi(s) \mathrm{d} s \leq k \int_{0}^{p(x, y)} \phi(s) \mathrm{d} s,
$$

for all $x, y \in X$. Then $\mathcal{T}$ has a unique fixed point.

Proof. Follows from Corollary 1 or Corollary 4 by taking $\psi(t)=t$ and

$$
t=\int_{0}^{t} \phi(s) \mathrm{d} s .
$$

Remark 3. Corollary 8 extends Theorem 2.1 of Branciari [10] from complete metric space to the setting of complete partial metric space.

\section{Illustrations}

Now we give some examples in support of our results.

Example 3. Let $X=\mathbb{R}$ and defined $p: X^{2} \rightarrow \mathbb{R}^{+}$by $p(x, y)=\max \{x, y\}$ for all $x, y \in X$. Then $p$ is a partial metric on $X$ and $(X, p)$ is a partial metric space. Let $\mathcal{T}: X \rightarrow X$ defined by $\mathcal{T}(x)=\frac{x}{3}$ and $\psi(t)=t, \phi(t)=\frac{t}{2}$ for all $t \geq 0$, where $\psi, \phi:[0, \infty) \rightarrow[0, \infty)$ are continuous and non-decreasing functions. Without loss of generality we assume that $x \geq y$. Then, choosing $x=1$ and $y=\frac{1}{2}$, we have

$$
\begin{gathered}
p(x, y)=\max \{x, y\}=x, \\
p(\mathcal{T} x, \mathcal{T} y)=\max \left\{\frac{x}{3}, \frac{y}{3}\right\}=\frac{x}{3}, \\
p(x, \mathcal{T} x)=\max \left\{x, \frac{x}{3}\right\}=x, \\
p(y, \mathcal{T} y)=\max \left\{y, \frac{y}{3}\right\}=y, \\
p(x, \mathcal{T} y)=\max \left\{x, \frac{y}{3}\right\}=x, \\
p(y, \mathcal{T} x)=\max \left\{y, \frac{x}{3}\right\}=y, \\
\Theta(x, y)=\max \left\{x, x, y, \frac{1}{3}(x+y+y)\right\}=x, \\
\theta(x, y)=\max \{x, y\}=x .
\end{gathered}
$$




\section{Result Analysis}

(1) We have

and

$$
\psi(p(\mathcal{T}(x), \mathcal{T}(y)))=\psi\left(\frac{x}{3}\right)=\frac{x}{3}
$$

$$
\psi(\Theta(x, y))-\phi(\theta(x, y))=\psi(x)-\phi(x)=x-\frac{x}{2} .
$$

Now consider inequality (4), we get

$$
\frac{x}{3} \leq x-\frac{x}{2}=\frac{x}{2}
$$

or

$$
\frac{1}{3} \leq \frac{1}{2}
$$

which true. Thus $\mathcal{T}$ satisfies all the hypothesis of Theorem 1 . Hence by applying Theorem $1, \mathcal{T}$ has a unique fixed point. It is seen that $0 \in X$ is the unique fixed point of $\mathcal{T}$.

(2) Now consider inequality (43), we get

$$
\frac{x}{3} \leq k \max \left\{x, x, y, \frac{1}{3}(x+y+y)\right\}=k x,
$$

or

$$
k \geq \frac{1}{3} .
$$

If we take $0<k<1$, then $\mathcal{T}$ satisfies all the hypothesis of Corollary 1 . Hence by applying Corollary $1, \mathcal{T}$ has a unique fixed point. It is seen that $0 \in X$ is the unique fixed point of $\mathcal{T}$.

(3) Now consider inequality (45), we have

$$
\frac{x}{3} \leq \alpha x+\beta x+\gamma y+\frac{\delta}{3}(x+2 y),
$$

putting $x=1$ and $y=\frac{1}{2}$, we get

$$
\frac{1}{3} \leq \alpha+\beta+\frac{1}{2} \gamma+\frac{2}{3} \delta
$$

The above inequality is satisfied for:

(i) $\alpha=\beta=\frac{1}{4}, \gamma=\frac{1}{3}$ and $\delta=0$, with $\alpha+\beta+\gamma+\delta=\frac{5}{6}<1$;

(ii) $\alpha=\frac{1}{6}, \beta=0, \gamma=\frac{1}{5}$ and $\delta=\frac{1}{4}$, with $\alpha+\beta+\gamma+\delta=\frac{13}{30}<1$;

(iii) $\alpha=0, \beta=\frac{1}{5}, \gamma=\frac{1}{4}$ and $\delta=\frac{1}{3}$, with $\alpha+\beta+\gamma+\delta=\frac{47}{60}<1$;

(iv) $\alpha=\frac{1}{5}, \beta=\frac{1}{4}, \gamma=0$ and $\delta=\frac{1}{7}$, with $\alpha+\beta+\gamma+\delta=\frac{83}{140}<1$.

Thus, $\mathcal{T}$ satisfies all the hypothesis of Corollary 3 . Hence by applying Corollary $3, \mathcal{T}$ has a unique fixed point. It is seen that $0 \in X$ is the unique fixed point of $\mathcal{T}$. 
Example 4. Let $X=\{1,2,3,4\}$ and $p: X \times X \rightarrow \mathbb{R}$ be defined by

$$
p(x, y)=\left\{\begin{array}{cl}
|x-y|+\max \{x, y\}, & \text { if } x \neq y, \\
x, & \text { if } x=y \neq 1, \\
0, & \text { if } x=y=1,
\end{array}\right.
$$

for all $x, y \in X$. Then $(X, p)$ is a complete partial metric space.

Define the mapping $\mathcal{T}: X \rightarrow X$ by

$$
\mathcal{T}(1)=1, \quad \mathcal{T}(2)=1, \quad \mathcal{T}(3)=2, \quad \mathcal{T}(4)=2 .
$$

Now, we have

$$
\begin{aligned}
& p(\mathcal{T}(1), \mathcal{T}(2))=p(1,1)=0 \leq \frac{3}{4} \cdot 3=\frac{3}{4} p(1,2), \\
& p(\mathcal{T}(1), \mathcal{T}(3))=p(1,2)=3 \leq \frac{3}{4} \cdot 5=\frac{3}{4} p(1,3), \\
& p(\mathcal{T}(1), \mathcal{T}(4))=p(1,2)=3 \leq \frac{3}{4} \cdot 7=\frac{3}{4} p(1,4), \\
& p(\mathcal{T}(2), \mathcal{T}(3))=p(1,2)=3 \leq \frac{3}{4} \cdot 4=\frac{3}{4} p(2,3), \\
& p(\mathcal{T}(2), \mathcal{T}(4))=p(1,2)=3 \leq \frac{3}{4} \cdot 6=\frac{3}{4} p(2,4), \\
& p(\mathcal{T}(3), \mathcal{T}(4))=p(2,2)=2 \leq \frac{3}{4} \cdot 5=\frac{3}{4} p(3,4) .
\end{aligned}
$$

Thus, $\mathcal{T}$ satisfies all the hypothesis of Corollary 2 with $k=\frac{3}{4}<1$. Now by applying Corollary 2, $\mathcal{T}$ has a unique fixed point, which in this case is 1 .

\section{Conclusion}

In this paper, we introduce two contractive conditions (i) generalized $(\psi-$ $\phi)$-weak contraction of type $(A)$ and (ii) generalized $(\psi-\phi)$-weak contraction of type $(B)$ in partial metric spaces and establish some fixed point results for above said contraction conditions in complete partial metric spaces. We support our results by some examples. Also, we obtain some fixed point results for mappings satisfying contractive conditions of integral type in partial metric spaces as corollaries to the results.

\section{Acknowledgement}

The author would like to thanks the anonymous referee for his/her careful reading and useful suggestions to improve the manuscript. 


\section{REFERENCES}

[1] M. Abbas and D. Doric, Common fixed point for generalized $(\psi-\phi)$-weak contractions, Mathematics, University of Niš, Serbia, 10 (2010), 1-10.

[2] M. Abbas and T. Nazir, Fixed point of generalized weakly contractive mappings in orpartial metric spaces, Fixed Point Theory and Applications, 2012 (2012), 1-19.

[3] T. Abdeljawad, E. Karapinar and K. Tas, Existence and uniqueness of common fixed point partial metric spaces, Applied Mathematics Letters, 24 (2011), 1900-1904.

[4] Ya. I. Alber and S. Guerre-Delabrieer, Principles of weakly contractive maps in Hilbert spaces, new results in operator theory, In: Gohberg I., Lyubich Y. (eds) New Results in Operator Theory and Its Applications. Operator Theory: Advances and Applications, Birkhauser Verlag, Basel 98 (1997), 7-22.

[5] I. Altun, F. Sola and H. Simsek, Generalized contractions on partial metric spaces, Topology and its Applications, 157 (2010), 2778-2785.

[6] I. Altun and A. Erduran, Fixed point theorems for monotone mappings on partial metric spaces, Fixed Point Theory and Applications, 2011 (2011), Article ID: 508730, 10 pages.

[7] H. Aydi, M. Abbas and C. Vetro, Partial Hausdorff metric and Nadler's fixed point theorem on partial metric spaces, Topology and its Applications, 159 (14) (2012), 3234-3242.

[8] S. Banach, Surles operation dans les ensembles abstraits et leur application aux equation integrals, Fundamenta Mathematicae, 3 (1922), 133-181.

[9] I. Beg and M. Abbas, Coincidence point and invariant approximation for mappings satisfying generalized weak contractive condition, Fixed Point Theory and Applications, 2006 (2006), Article ID: 74503, 7 pages.

[10] A. Branciari, A fixed point theorem for mapping satisfying a general contractive condition of integral type, International Journal of Mathematics and Mathematical Sciences, 29 (2002), 531-536.

[11] R. P. Chi, E. Karapinar and T. D. Thanh, A generalized contraction principle in partial metric spaces, Mathematical and Computer Modelling, 55 (2012), 1673-1681.

[12] L. B. Cirić, B. Samet, H. Ayadi and C. Vetro, Common fixed point of generalized contraction on partial metric spaces with applications, Applied Mathematics and Computation, 218 (2011), 2398-2406.

[13] B. S. Choudhury, P. Konar, B. E. Rhoades and N. Metiya, Fixed point theorems for generalized weakly contractmapping, Nonlinear Analysis, 74 (6) (2011), 2116-2126.

[14] B. S. Choudhury, N. Metiya and M. Postolache, A generalized weak contraction principle with applications to coupled coincidence point problems, Fixed Point Theory and Application, 2013 (2013), Article ID: 152.

[15] D. Doric, Common fixed point for generalized $(\psi, \varphi)$-weak contractions, Applied Mathematics Letters, 22 (2009), 1896-1900.

[16] P. N. Dutta and B. S. Choudhury, A generalisation of contraction principle in metric spaces, Fixed Point Theory and Applications, 2008 (2008), Article ID: 406368, 8 pages. 
[17] E. Karapinar and U. Yüksel, Some common fixed point theorems in partial metric space, Journal of Applied Mathematics, 2011 (2011), Article ID: 263621.

[18] Z. Kadelburg, H. K. Nashine and S. Radenovic, Fixed point results under various contractive conditions in partial metric spaces, Revista de la Real Academia de Ciencias Exactas, Físicas y Naturales, Serie A Matemáticas (RACSAM), 107 (2013), 241-256.

[19] M. S. Khan, M. Swaleh and S. Sessa, Fixed point theorems by altering distance between the points, Bulletin of the Australian Mathematical Society, 30 (1) (1984), 1-9.

[20] H. P. A. Künzi, Nonsymmetric distances and their associated topologies about the origins of basic ideas in the area of asymptotic topology, Handbook of the History General Topology (eds. C.E. Aull and R. Lowen), Springer Science+Business Media B.V., 3 (2001), 853-868.

[21] S. G. Matthews, Partial metric topology, Research report 2012, Department of Computer Science, University of Warwick, 1992.

[22] S. G. Matthews, Partial metric topology, Proceedings of the 8th summer conference on topology and its applications, Annals of the New York Academy of Sciences, 728 (1994), 183-197.

[23] P. P. Murthy, K. Tas and Uma Devi Patel, Common fixed point theorems for generalized $(\phi, \psi)$-weak contraction, Journal of Inequalities and Applications, 2015 (2015), Article ID: 139, 14 pages.

[24] H. K. Nashine, Z. Kadelburg, S. Radenovic and J. K. Kim, Fixed point theorems under Hardy-Rogers contractive conditions on 0-complete ordered partial metric spaces, Fixed Point Theory and Applications, 2012 (2012), 1-15.

[25] O. Popescu, Fixed points for $(\psi, \phi)$-weak contractions, Applied Mathematics Letters, 24 (1) (2011), 1-4.

[26] B. E. Rhoades, Some theorems on weakly contractive maps, Nonlinear Analysis, Theory, Methods and Applications, 47 (4) (2001), 2683-2693.

[27] M. Schellekens, A characterization of partial metrizibility: domains are quantifiable, Theoritical Computer Science, 305 (1-3) (2003), 409-432.

[28] W. Shatanawi and M. Postolache, Coincidence and fixed point results for generalized weak contractions in the sense of Berinde on partial metric spaces, Fixed Point Theory and Applications, 2013 (2013), Article ID: 54.

[29] Q. Zhang and Y. Song, Fixed point theory for generalized $\varphi$-weak contractions, Applied Mathematics Letters, 22 (2009), 75-78.

[30] O. Vetro, On Banach fixed point theorems for partial metric spaces, Applied General Topology, 6 (12) (2005), 229-240.

[31] P. Waszkiewicz, Partial metrizibility of continuous posets, Mathematical Structures in Computer Science, 16 (2) (2006), 359-372.

\section{G.S. SAluJA}

Department of Mathematics

Govt. Kaktiya P.G. College, Jagdalpur

JAGDALPUR - 494001 (C.G.)

INDIA

E-mail address: saluja1963@gmail.com 